\title{
Convex and concave types of second baroclinic mode internal solitary waves
}

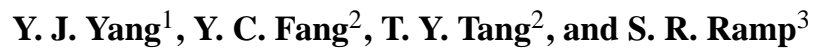 \\ ${ }^{1}$ Department of Marine Science, Naval Academy, Kaohsiung, Taiwan \\ ${ }^{2}$ Institute of Oceanography, National Taiwan University, Taipei, Taiwan \\ ${ }^{3}$ Monterey Bay Aquarium Research Institute, Moss Landing, California, USA \\ Received: 9 August 2010 - Revised: 11 October 2010 - Accepted: 12 October 2010 - Published: 2 November 2010
}

\begin{abstract}
Two types of second baroclinic mode (mode-2) internal solitary waves (ISWs) were found on the continental slope of the northern South China Sea. The convex waveform displaced the thermal structure upward in the upper layer and downward in the lower layer, causing a bulge in the thermocline. The concave waveform did the opposite, causing a constriction. A few concave waves were observed in the South China Sea, marking the first documentation of such waves. On the basis of the Korteweg-de Vries (K-dV) equation, an analytical three-layer ocean model was used to study the characteristics of the two mode-2 ISW types. The analytical solution was primarily a function of the thickness of each layer and the density difference between the layers. Middle-layer thickness plays a key role in the resulting mode-2 ISW. A convex wave was generated when the middle-layer thickness was relatively thinner than the upper and lower layers, whereas only a concave wave could be produced when the middle-layer thickness was greater than half the water depth. In accordance with the K-dV equation, a positive and negative quadratic nonlinearity coefficient, $\alpha_{2}$, which is primarily dominated by the middle-layer thickness, resulted in convex and concave waves, respectively. The analytical solution showed that the wave propagation of a convex (concave) wave has the same direction as the current velocity in the middle (upper or lower) layer. Analysis of the three-layer ocean model properly reproduced the characteristics of the observed mode- 2 ISWs in the South China Sea and provided a criterion for the existence of convex or concave waves. Concave waves were seldom seen because of the rarity of a stratified ocean with a thick middle layer. This analytical result agreed well with the observations.
\end{abstract}

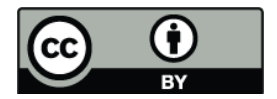

Correspondence to: Y. J. Yang (yjyang@cna.edu.tw)

\section{Introduction}

An internal solitary wave (ISW) is a commonly observed phenomenon in many of the world's stratified oceans. Its prevalent form is the first baroclinic mode (mode-1) ISW. A mode-1 ISW produces strong vertical mixing and can impact underwater vessels or structures. Mode-1 ISWs have been widely investigated through theoretical, modeling and observational efforts (Helfrich and Melville, 2006; Apel et al., 2007).

The second baroclinic mode (mode-2) ISW is much less common than the mode-1 ISW and has only occasionally been observed. Theoretically, a mode-2 ISW should take one of two types of waveforms. In the upper layer, a "convex wave" has a leading upward displacement of an isotherm ahead of the wave and a trailing downward displacement behind it. Deeper in the water column, the opposite displacement occurs, creating what has been described as a bulge-shaped, double-humped, varicose, or sausage-type wave (Davis and Acrivos, 1967; Stamp and Jacka, 1995; Ostrovsky and Stepanyants, 2005; Moum et al., 2008). Pioneering investigations have described this type of wave, including theoretical investigations (Benjamin, 1967; Davis and Acrivos, 1967; Akylas and Grimshaw, 1992; Vlasenko, 1994; Grimshaw, 1997), laboratory experiments (Davis and Acrivos, 1967; Kao and Pao, 1980; Maxworthy, 1980; Honji et al., 1995; Stamp and Jacka, 1995; Vlasenko and Hutter, 2001; Mehta et al., 2002; Sutherland, 2002), numerical analyses (Tung et al., 1982; Terez and Knio, 1998; Rubino et al., 2001; Vlasenko and Hutter, 2001; Rusås and Grue, 2002; Stastna and Peltier, 2005; Vlasenko and Alpers, 2005), and field observations (Farmer and Smith, 1980; Konyaev et al., 1995; Saggio and Imberger, 1998, 2001; Antenucci et al., 2000; Boegman et al., 2003; Duda et al., 2004; Yang et al., 2004; Bougucki et al., 2005; Sabinin and Serebryany, 2005; Moum et al., 2008; Shroyer et al., 2010). 


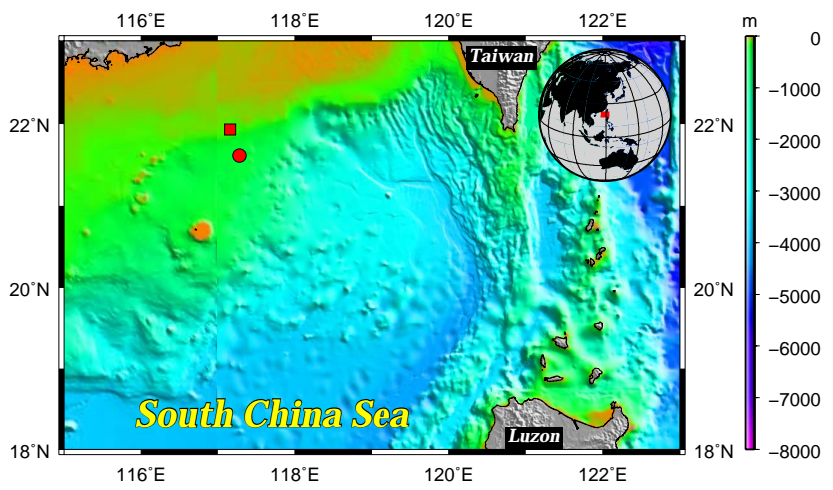

Fig. 1. The mooring location (shown as a red dot) on the continental slope of the northern South China Sea. The local water depth was $350 \mathrm{~m}$. The square marks the location of the density profile in Fig. 4.

Theoretically, however, a second type of mode- 2 wave is also possible. Sometimes called "reverse convex" waves, these waves are referred to here as "concave" waves and have the opposite character from convex waves. The concave waves have an hourglass-shaped constriction of the thermal structure with downward followed by upward deflection of the thermal structure in the upper layer associated with the opposite condition in the lower layer.

A long-term current velocity and temperature mooring was deployed on the continental slope of the northern South China Sea (Fig. 1) under the joint research program known as Variations Around the Northern South China Sea (VANS, supported by Taiwan) and the Windy Islands Soliton Experiment (WISE, supported by the United States). The observed current velocity and temperature data revealed that mode-2 ISWs were active, and both convex and concave waves were found. The project identified 78 convex waves (Yang et al., 2009) and four concave waves, marking the first time concave waves have been observed in nature.

This article describes the characteristics of two distinct types of mode-2 ISWs on the continental slope of the northern South China Sea and discusses how stratification affects their formation. Furthermore, an analytical threelayer ocean model was used to study and interpret the observed convex and concave waves. The remainder of this paper is organized as follows. Section 2 describes the observational results of the convex and concave waves. The analytical interpretations applied for mode-2 ISWs under a three-layer ocean model are presented, and the results are shown in Sects. 3 and 4, respectively. Finally, Sect. 5 provides a discussion of the two mode-2 ISW types.

\section{Observations}

The current velocity and temperature mooring was deployed at a depth of $350 \mathrm{~m}$ from 29 April to 28 July 2005 and from 2 November 2005 to 24 February 2006. A 300-kHz self-contained acoustic Doppler current profiler (ADCP) was mounted at $100 \mathrm{~m}$ and measured the current velocity from $15 \mathrm{~m}$ to $95 \mathrm{~m}$ in 4-m bins and recorded the temperature at $100 \mathrm{~m}$. The ping rate was $1 \mathrm{~s}$, and the averaging and recording interval was $1 \mathrm{~min}$. Temperature was also recorded every minute. Three ducted paddlewheel recording current meters (RCM8s) were located at $160 \mathrm{~m}, 220 \mathrm{~m}$, and $310 \mathrm{~m}$. The RCM8s recorded the current velocity and temperature every $5 \mathrm{~min}$. The mooring had 16 temperature recorders (one ADCP, three RCM8s, seven temperature-pressure recorders - TPs, four conductivity-temperature-depth sensors - CTDs, and one temperature recorder - T-pod) spanning depths from $32 \mathrm{~m}$ to $338 \mathrm{~m}$. The TPs and T-pod sampled every minute, and the CTDs sampled every 2 min.

Because the passage of ISWs would induce a temperature perturbation, a time series of temperature measurements was used to identify mode- 2 ISW episodes. In a convex wave, the temperature theoretically undergoes a decreasing and then an increasing temperature evolution in the upper layer and the opposite temperature evolution in the lower layer. A concave wave has reverse temperature evolutions; therefore, for convex and concave waves, the isotherm contour plot would reveal a "double-humped" and a "reverse double-humped" waveform, respectively. Whether the wave was convex or concave could be determined by the thermal displacement pattern. During the observation period, 78 convex waves and four concave waves were identified.

Current velocity was also used to further confirm the mode-2 ISW episode. Theoretically, the mode-2 ISW current velocity has a three-layered structure with two vertical nodal points (zero-crossing points). The current velocities of the upper and lower layers travel in the same direction but opposite to the current in the middle layer. The convexwave propagation direction is consistent with the current direction in the middle layer. The propagation direction of a concave wave is unknown, and the relationship between current distribution and propagation direction has not been studied. For each mode-2 ISW episode, the horizontal current velocity perturbation, which could be associated with the mode- 2 ISW, was calculated by subtracting the background current velocity from the measured current velocity. The background current was defined as the average current $30 \mathrm{~min}$ before the episode.

The vertical velocity perturbation $\left(w^{\prime}\right)$ of the whole water column associated with mode-2 ISWs was estimated from the wave temperature perturbation using the temperature conservation equation with the assumption of negligible horizontal advection and heat conduction:

$w^{\prime}(z, t) \approx-\frac{\frac{T^{\mathrm{h}}(z, t+\Delta t)-T^{\mathrm{h}}(z, t)}{\Delta t}}{\frac{T^{\mathrm{b}}(z+\Delta z, t)-T^{\mathrm{b}}(z, t)}{\Delta z}}$,

where $T^{\mathrm{h}}(z, t)$ is the 5-h high-pass-filtered temperature, $T^{\mathrm{b}}(z, t)$ is the $36-\mathrm{h}$ low-pass-filtered temperature, $z$ is the depth where the temperature sensor was mounted, $\Delta z$ is the depth difference between the two temperature sensors, 
(a) Temperature $\left({ }^{\circ} \mathrm{C}\right)$

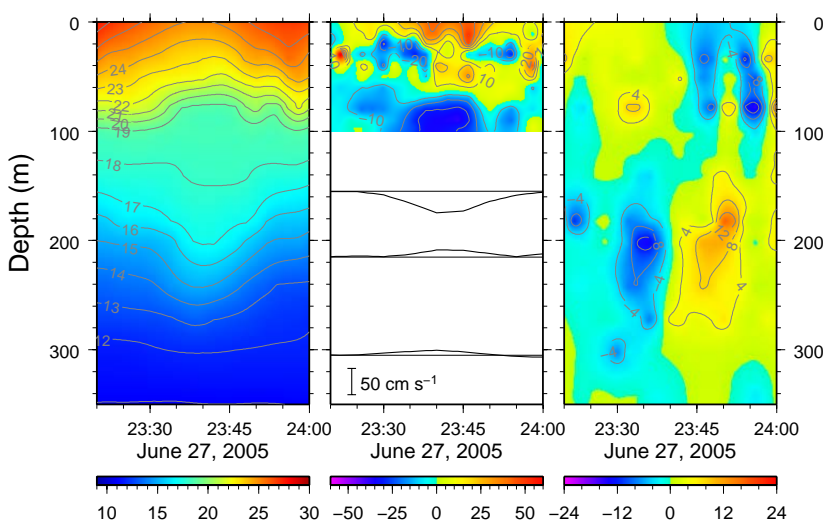

Fig. 2. The left (a), middle (b), and right (c) panels represent the contours of isotherms, perturbations of the eastward current component $\left(u^{\prime}\right)$, and vertical current component $\left(w^{\prime}\right)$ as functions of time and depth, respectively, from 23:20 to 24:00 UTC on 27 June 2005. The temperature data were measured by 16 temperature sensors at depths from 32 to $338 \mathrm{~m}$. The eastward current data were measured by an ADCP between $15 \mathrm{~m}$ and $95 \mathrm{~m}$ and three RCM8s at $160 \mathrm{~m}, 220 \mathrm{~m}$, and $310 \mathrm{~m}$. The contour intervals are $1^{\circ} \mathrm{C}, 10 \mathrm{~cm} \mathrm{~s}^{-1}$, and $4 \mathrm{cms}^{-1}$ for temperature, $u^{\prime}$, and $w^{\prime}$, respectively. Positive $u^{\prime}$ and $w^{\prime}$ indicate eastward and upward velocities, respectively.

$t$ is the time when the mode- 2 ISW passed the mooring, and $\Delta t$ is $1 \mathrm{~min}$. Theoretically, $w^{\prime}$ has a two-layered structure with one nodal point vertically and has opposite directions in the upper and lower layers, respectively. In the upper layer, $w^{\prime}$ is positive and then negative (upwelling/downwelling) for convex waves and negative and then positive (downwelling/upwelling) for concave waves. The values of $w^{\prime}$ computed in this way agreed well with the ADCP observations where available (in the upper water column, <100 m depth).

In the following sections, characteristics of a convex wave and a concave wave are illustrated.

\subsection{Convex waves}

Characteristics of convex waves such as amplitude, time scale, characteristic width, and phase speed have been reported by Yang et al. (2009). A typical isotherm contour (Fig. 2a) behaved like a "double-humped" waveform from 23:20 to 24:00 UTC on 27 June 2005, indicating a convex wave. Figure $2 \mathrm{~b}$ shows the perturbation of the eastward velocity component $\left(u^{\prime}\right)$. The $u^{\prime}$ induced by the convex wave had an eastward acceleration and then a deceleration evolution above $60 \mathrm{~m}$ and at $220 \mathrm{~m}$ and $310 \mathrm{~m}$, and the opposite pattern between $60 \mathrm{~m}$ and $100 \mathrm{~m}$ and at $160 \mathrm{~m}$. This result implies that there were two $u^{\prime}$ nodal points: one at $\sim 60 \mathrm{~m}$ and another between $160 \mathrm{~m}$ and $220 \mathrm{~m}$. The perturbation of the northward velocity component $\left(v^{\prime}\right.$, not shown) had an evolution similar to $u^{\prime}$, whereas the $v^{\prime}$ (a) Temperature $\left({ }^{\circ} \mathrm{C}\right)$

(b) $u^{\prime}\left(\mathrm{cm} \mathrm{s}^{-1}\right)$

(c) $W^{\prime}\left(\mathrm{cm} \mathrm{s}^{-1}\right)$

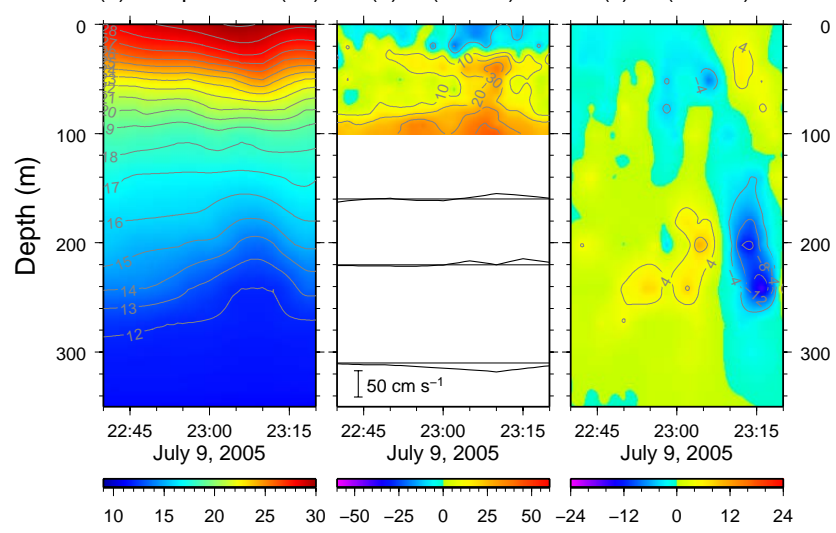

Fig. 3. Same as Fig. 2 except from $22: 40$ to $23: 20$ UTC on 9 July 2005.

amplitude was much smaller than that of $u^{\prime}$. Therefore, the primary propagation direction of this convex wave was westward, consistent with mode- 1 waves observed in the area (Ramp et al., 2010). The corresponding depths of the local maximum and minimum $w^{\prime}$ were near $80 \mathrm{~m}$ and $200 \mathrm{~m}$, respectively (Fig. 2c). The positions of the local maximum/minimum $w^{\prime}$ were close to the positions of the two $u^{\prime}$ nodal points.

\subsection{Concave waves}

An example of a concave wave is shown in Fig. 3. The thermal displacement pattern (Fig. 3a) of the concave wave was opposite that of the convex wave. The vertical isotherm displacements at $24^{\circ} \mathrm{C}$ and $15^{\circ} \mathrm{C}$ were approximately $-20 \mathrm{~m}$ and $40 \mathrm{~m}$, respectively. The isotherms showed a downward and then an upward displacement above $140 \mathrm{~m}$ and the opposite evolution below, in which a "reverse doublehumped" waveform evolved in the isotherm depth contour. Similar to the previous convex wave, the $v^{\prime}$ amplitude was relatively smaller than that of $u^{\prime}$; hence we only consider $u^{\prime}$ here. Figure $3 \mathrm{~b}$ demonstrates how $u^{\prime}$ was induced by the concave wave. At 23:10 UTC on 9 July 2005, a negative $u^{\prime}$ was found above $30 \mathrm{~m}$, whereas a positive $u^{\prime}$ was found below. The $u^{\prime}$ nodal point was identified near $30 \mathrm{~m}$. The RCM8s at $160 \mathrm{~m}$ and $220 \mathrm{~m}$ also experienced a positive $u^{\prime}$ between $30 \mathrm{~m}$ and $220 \mathrm{~m}$, whereas the RCM8 at $310 \mathrm{~m}$ experienced a negative $u^{\prime}$. This result shows that another $u^{\prime}$ nodal point was located between $220 \mathrm{~m}$ and $310 \mathrm{~m}$. The vertical velocity component, $w^{\prime}$, also revealed a clear modal structure (Fig. 3c). At 23:05 UTC on 9 July 2005, a downwelling occurred above $140 \mathrm{~m}$, whereas an upwelling occurred below $140 \mathrm{~m}$. Consequently, the $w^{\prime}$ nodal point was near $140 \mathrm{~m}$. The perturbation induced by the concave wave was a downwelling/upwelling process above and below the $w^{\prime}$ nodal point. The corresponding depths of the local maximum and minimum $w^{\prime}$ were near $30 \mathrm{~m}$ and $240 \mathrm{~m}$, 


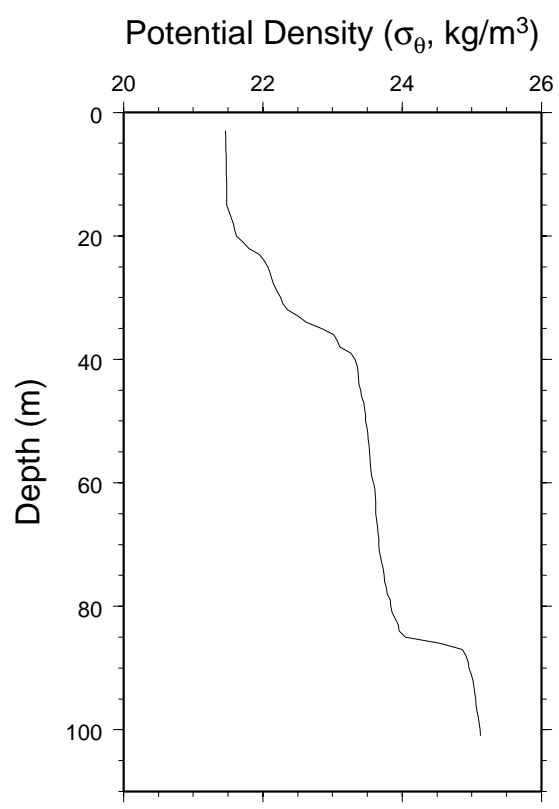

Fig. 4. Vertical profile of density at 22:56 UTC on 20 May 2001. The data were obtained at $21^{\circ} 55.8^{\prime} \mathrm{N}, 117^{\circ} 9.6^{\prime} \mathrm{E}$ (shown in Fig. 1), where the local water depth was $108 \mathrm{~m}$.

respectively. The positions of the local maximum/minimum $w^{\prime}$ were also in accordance with the positions of the two $u^{\prime}$ nodal points.

The profile of this example was asymmetrical, and the temperature isopleths (Fig. 3a) were more or less flat in the front and rear of the wave, respectively. The vertical velocity (Fig. 3c) also illustrated this phenomenon. This example was slightly different from the previous convex wave example. The asymmetrical profile of the thermal structure of the wave could have been due to interference by other internal waves or by background current.

Only four concave waves were found. The average amplitude of the upper part of the wave was $15 \pm 4.3 \mathrm{~m}$, while it was $27 \pm 12.1 \mathrm{~m}$ for the lower part. The average time duration was $29 \pm 4.9$ min.

Yih (1960) inferred that a three-layer fluid system could have mode-2 waves. Actually, a nearly three-layer density structure has been observed (Fig. 4) around the study area. Therefore, an analytic three-layer ocean model was established to study the characteristics of convex and concave waves.

\section{Analytic three-layer ocean model}

The vertical velocity structure function, $W_{n}$, is governed by the Taylor-Goldstein equation and was used to consider the hydrostatic, frictionless internal motion without background current and to satisfy the Boussinesq approximation (Gill, 1982): $\frac{d^{2} W_{n}(z)}{d z^{2}}+\frac{N^{2}(z)}{c_{n}^{2}} W_{n}(z)=0$,

where $W_{n}(z)$ is the eigenfunction (or vertical structure function) for the $n$-th mode, $c_{n}$ is the eigenvalue (or modal phase velocity of the linear wave), and $N(z)$ is the BruntVäisälä (or buoyancy) frequency. The vertical modes of horizontal $U_{n}(z)$ and vertical motion are related by $U_{n}(z)=$ $d W_{n}(z) / d z$. The theoretical vertical structures can be calculated by the buoyancy frequency profile.

For the simplified case of a three-layer ocean (Fig. 5a), the thicknesses corresponding to the upper, middle, and lower layers are $h_{1}, h_{2}$, and $h_{3}$, respectively. The densities corresponding to the upper, middle, and lower layers are $\rho_{0}-\Delta \rho_{1}, \rho_{0}$, and $\rho_{0}+\Delta \rho_{2}$, respectively, where $0<\Delta \rho_{1}$, $\Delta \rho_{2} \ll \rho_{0} . \quad N(z)=0$ except at two interfaces, $N\left(-h_{1}\right)=$ $\sqrt{g_{1}^{\prime} / \Delta z}$ and $N\left(-h_{1}-h_{2}\right)=\sqrt{g_{2}^{\prime} / \Delta z}$, where $g_{1}^{\prime}$ and $g_{2}^{\prime}$ are $\Delta \rho_{1} g / \rho_{0}$ and $\Delta \rho_{2} g / \rho_{0}$, respectively, $g$ is gravitational acceleration, and $\Delta z$ is a small value variable.

Imposed on the rigid-lid boundary condition, $W_{n}(0)=0$ and $W_{n}\left(-h_{1}-h_{2}-h_{3}\right)=0$, the analytical solutions for Eq. (2) are as follows:

$$
\begin{aligned}
& W_{n}(z) \\
& =\left\{\begin{array}{lr}
-\frac{z}{h_{1}} & -h_{1} \leq z \leq 0 \\
\frac{1-\gamma_{n}}{h_{2}}\left(z+h_{1}+h_{2}\right)+\gamma_{n} & -h_{1}-h_{2} \leq z \leq-h_{1} \\
\frac{\gamma_{n}}{h_{3}}\left(z+h_{1}+h_{2}+h_{3}\right) & -h_{1}-h_{2}-h_{3} \leq z \leq-h_{1}-h_{2}
\end{array}\right.
\end{aligned}
$$

$$
\begin{aligned}
& U_{n}(z) \\
& =\left\{\begin{array}{cc}
-\frac{1}{h_{1}} & -h_{1} \leq z \leq 0 \\
\frac{1-\gamma_{n}}{h_{2}} & -h_{1}-h_{2} \leq z \leq-h_{1} \\
\frac{\gamma_{n}}{h_{3}} & -h_{1}-h_{2}-h_{3} \leq z \leq-h_{1}-h_{2}
\end{array}\right.
\end{aligned}
$$

where $\gamma_{n}$ is the ratio of the vertical velocity component at the lower interface to that at the upper interface: $W_{n}\left(-h_{1}-h_{2}\right) / W_{n}\left(-h_{1}\right)$. Integrating Eq. (2) from $-h_{1}-$ $\Delta z / 2$ to $-h_{1}+\Delta z / 2$ and from $-h_{1}-h_{2}-\Delta z / 2$ to $-h_{1}-$ $h_{2}+\Delta z / 2, \gamma_{n}$ can be expressed as follows:

$$
\begin{aligned}
& \gamma_{n}=-\left(\frac{h_{2} g_{1}^{\prime}}{c_{n}^{2}}-\frac{h_{2}}{h_{1}}-1\right) \\
& \gamma_{n}=-\left(\frac{h_{2} g_{2}^{\prime}}{c_{n}^{2}}-\frac{h_{2}}{h_{3}}-1\right)^{-1}
\end{aligned}
$$

Substituting $\gamma_{n}$ of Eq. (6) into (5), a single phase velocity equation $c_{n}$ is obtained:

$$
\begin{aligned}
& \left(h_{1}+h_{2}+h_{3}\right) c_{n}^{4}-\left[h_{1}\left(h_{2}+h_{3}\right) g_{1}^{\prime}+\left(h_{1}+h_{2}\right) h_{3} g_{2}^{\prime}\right] c_{n}^{2} \\
& +h_{1} h_{2} h_{3} g_{1}^{\prime} g_{2}^{\prime}=0
\end{aligned}
$$



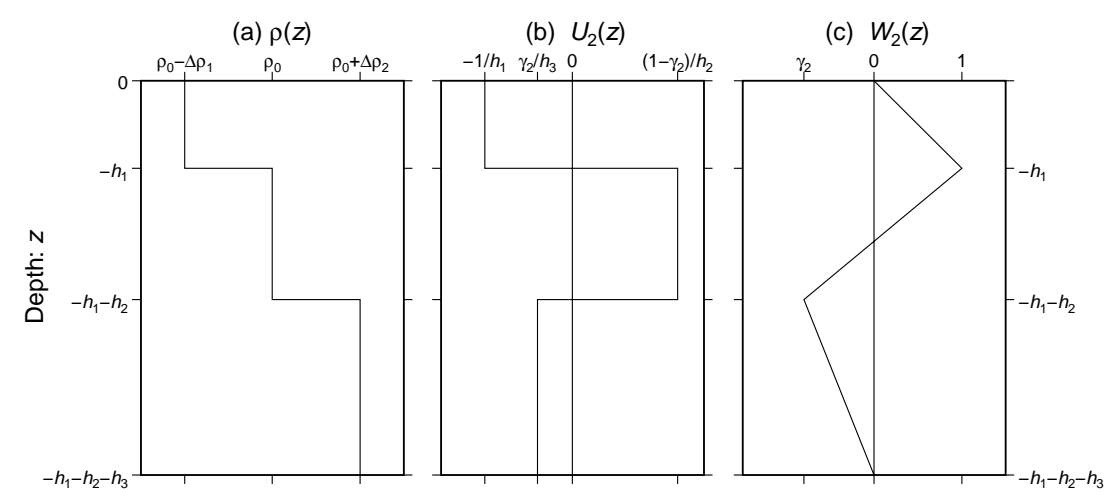

Fig. 5. The left (a), middle (b), and right (c) panels represent the mode-2 ISW vertical density profile $(\rho)$ and vertical structure functions of horizontal $\left(U_{2}\right)$ and vertical $\left(W_{2}\right)$ current components, respectively, in the three-layer rigid-lid ocean.

Therefore, the eigenvalues (or linear internal wave modal phase speed) of the Taylor-Goldstein equation, $c_{1}$ and $c_{2}$, are as follows:

$$
\begin{aligned}
c_{1}^{2} & =\frac{h_{1}\left(h_{2}+h_{3}\right) g_{1}^{\prime}+\left(h_{1}+h_{2}\right) h_{3} g_{2}^{\prime}}{2\left(h_{1}+h_{2}+h_{3}\right)} \\
& +\frac{\sqrt{\left(h_{1}\left(h_{2}+h_{3}\right) g_{1}^{\prime}+\left(h_{1}+h_{2}\right) h_{3} g_{2}^{\prime}\right)^{2}-4\left(h_{1}+h_{2}+h_{3}\right) h_{1} h_{2} h_{3} g_{1}^{\prime} g_{2}^{\prime}}}{2\left(h_{1}+h_{2}+h_{3}\right)}
\end{aligned}
$$

$$
\begin{aligned}
c_{2}^{2} & =\frac{h_{1}\left(h_{2}+h_{3}\right) g_{1}^{\prime}+\left(h_{1}+h_{2}\right) h_{3} g_{2}^{\prime}}{2\left(h_{1}+h_{2}+h_{3}\right)} \\
& -\frac{\sqrt{\left(h_{1}\left(h_{2}+h_{3}\right) g_{1}^{\prime}+\left(h_{1}+h_{2}\right) h_{3} g_{2}^{\prime}\right)^{2}-4\left(h_{1}+h_{2}+h_{3}\right) h_{1} h_{2} h_{3} g_{1}^{\prime} g_{2}^{\prime}}}{2\left(h_{1}+h_{2}+h_{3}\right)}
\end{aligned}
$$

where $c_{1}$ and $c_{2}$, which have similar formulations derived by Grimshaw et al. (1997) and Rubino et al. (2001), represent the phase speed of faster (mode-1) and slower (mode-2) mode linear internal waves, respectively. If there is no density difference at either the upper $\left(\Delta \rho_{1}=\right.$ $\left.0, \Delta \rho_{2} \neq 0\right)$ or lower $\left(\Delta \rho_{1} \neq 0, \Delta \rho_{2}=0\right)$ interfaces, the three-layer ocean model degenerates into a two-layer ocean model and only mode- 1 motion remains, such that $c_{2}=0$ and $c_{1}=\sqrt{h_{1}\left(h_{2}+h_{3}\right) g_{1}^{\prime} /\left(h_{1}+\left(h_{2}+h_{3}\right)\right)}$ or $c_{1}=\sqrt{\left(h_{1}+h_{2}\right) h_{3} g_{2}^{\prime} /\left(\left(h_{1}+h_{2}\right)+h_{3}\right)}$, respectively.

From Eqs. (5) and (6), we can obtain the relationship between $\gamma_{1}$ and $\gamma_{2}: \gamma_{1}-\gamma_{2}=\left(c_{2}^{-2}-c_{1}^{-2}\right) h_{2} g_{1}^{\prime}>0$ and $\gamma_{1} \gamma_{2}=-g_{1}^{\prime} / g_{2}^{\prime}<0$. Thus, $\gamma_{1}>0$ and $\gamma_{2}<0$. For $n=1$, the mode- 1 motion accompanies faster phase speed $\left(c_{1}\right)$ and in-phase vertical motion at the upper and lower interfaces $\left(\gamma_{1}>0\right)$. However, if $n=2$, the mode- 2 motion accompanies the slower phase speed $\left(c_{2}\right)$ and out-of-phase vertical motion at the upper and lower interfaces $\left(\gamma_{2}<0\right)$. The second-mode eigenfunctions of the Taylor-Goldstein equation, $U_{2}(z)$ and $W_{2}(z)$, are shown in Fig. $5 \mathrm{~b}$ and c, respectively.
According to Benney (1966), Lee and Beardsley (1974), Pelinovsky and Shavratsky (1977), Maslowe and Redekopp (1980), Grimshaw (1981), Gear and Grimshaw (1983), Apel et al. (1997), and Apel (2003), the modal displacement $\eta_{n}$ is governed by the Kortewegde Vries (K-dV) equation neglecting rotational effects and energy exchange between modes and assuming weakly nonlinear finite-amplitude plane-progressive waves propagating in a specific direction:

$\frac{\partial \eta_{n}}{\partial t}+c_{n} \frac{\partial \eta_{n}}{\partial x}+\alpha_{n} \eta_{n} \frac{\partial \eta_{n}}{\partial x}+\beta_{n} \frac{\partial^{3} \eta_{n}}{\partial x^{3}}=0$,

where $x$ is the wave front propagation direction and $\alpha_{n}$ and $\beta_{n}$ are the quadratic nonlinearity and dispersive coefficients for the $n$-th mode, respectively. Both coefficients are also called "environmental parameters," because they account for conditions such as stratification (or Taylor-Goldstein equation eigenfunctions) and water depth $(H)$ without background current as follows (Benney, 1966; Lee and Beardsley, 1974):

$$
\begin{gathered}
\alpha_{n}=\frac{3 c_{n}}{2} \frac{\int_{-H}^{0}\left(d W_{n} / d z\right)^{3} d z}{\int_{-H}^{0}\left(d W_{n} / d z\right)^{2} d z} \\
\beta_{n}=\frac{c_{n}}{2} \frac{\int_{-H}^{0} W_{n}^{2} d z}{\int_{-H}^{0}\left(d W_{n} / d z\right)^{2} d z}
\end{gathered}
$$

If the shallow-water approximation holds, the analytical solution for the $\mathrm{K}-\mathrm{dV}$ equation is a squared hyperbolic secant function: $\eta_{n}=\eta_{0, n} \operatorname{sech}^{2}\left(\left(x-v_{n} t\right) / \Delta_{n}\right)$. Here, $\eta_{0, n}$ is the amplitude, $v_{n}=c_{n}+\left(\alpha_{n} \eta_{0, n} / 3\right)$ is the nonlinear phase speed, and $\Delta_{n}=\sqrt{12 \beta_{n} / \alpha_{n} \eta_{0, n}}$ is the nonlinear characteristic width; $\eta_{0, n}$ or $v_{n}$ increases when $\Delta_{n}$ decreases. The signs of $\alpha_{n}$ and $\eta_{0, n}$ are the same (Apel et al., 1997).

Consequently, amplitude $\left(A_{n}\right)$, vertical $\left(w_{n}\right)$ and horizontal $\left(u_{n}\right)$ current velocity components, and the quadratic nonlinearity coefficient $\left(\alpha_{n}\right)$ can be calculated from Eqs. (3), 
(4), and (11) for mode-1 $(n=1)$ and mode-2 $(n=2)$ ISWs in this three-layer ocean model:

$$
\begin{aligned}
& A_{n}(x, z, t) \\
& =\eta_{n}(x, t) W_{n}(z)=\eta_{0, n} W_{n}(z) \operatorname{sech}^{2}\left(\frac{x-v_{n} t}{\Delta_{n}}\right) \\
& w_{n}(x, z, t)=\frac{\partial A_{n}(x, z, t)}{\partial t} \\
& =\frac{2 v_{n}}{\Delta_{n}} \eta_{0, n} W_{n}(z) \operatorname{sech}^{2}\left(\frac{x-v_{n} t}{\Delta_{n}}\right) \tanh \left(\frac{x-v_{n} t}{\Delta_{n}}\right) \\
& u_{n}(x, z, t) \\
& =-\int \frac{\partial w_{n}}{\partial z} d x=v_{n} \eta_{0, n} U_{n}(z) \operatorname{sech}^{2}\left(\frac{x-v_{n} t}{\Delta_{n}}\right) \\
& \alpha_{n}=\frac{3 c_{n}}{2} \frac{\frac{\gamma_{n}^{3}}{h_{3}^{2}}+\frac{\left(1-\gamma_{n}\right)^{3}}{h_{2}^{2}}-\frac{1}{h_{1}^{2}}}{h_{3}^{2}}+\frac{\left(1-\gamma_{n}\right)^{2}}{h_{2}}+\frac{1}{h_{1}}
\end{aligned}
$$

By Eq. (16), the quadratic nonlinearity coefficient is not only a function of layered thickness but also of density difference. It is more complicated than the two-layer ocean model solution. The signs of $\eta_{0, n}$ (or $\alpha_{n}$ ) represent the polarities of ISWs, with positive values for the elevation type of mode-1 and convex type of mode-2 ISWs, and negative values for the depression type of mode- 1 and concave type of mode-2 ISWs.

Physically, each interface could have displacement with two degrees of freedom so that a three-layer ocean (two interfaces) could have the following four types of ISWs: (1) in-phase motion in the upper and lower interfaces; both have upward and then downward interfacial displacement, which corresponds to the elevation type of mode-1 ISW; (2) in-phase motion of the upper and lower interfaces; both have downward and then interfacial upward displacement, which corresponds to the depression type of mode-1 ISW, (3) out-of-phase motion in upper and lower interfaces; the upper interface produces upward and then downward displacement and the lower interface evolves as downward and then upward displacement, which corresponds to the convex type of mode-2 ISW; and (4) out-of-phase motion in the upper and lower interfaces; if the upper interface produces downward and then upward displacement and the lower interface evolves as upward and then downward displacement, this corresponds to a concave type of mode- 2 ISW. In summary, there are two vertical modes in the threelayer ocean model. Each ISW mode has two polarities: elevation and depression types for mode-1 ISW and convex and concave types for mode- 2 ISW. The analytical results of the three-layer ocean model agree with the physical explanation.

The convex and concave wave solutions are discussed in the next section. Meanwhile, the mode-2 ISW polarity will be examined by changing the layer thickness and density.

\section{Results}

Two cases are examined for mode-2 ISW solutions. According to Eqs. (5), (9), and (16), the solutions and polarity of mode-2 ISWs depend on the sign of $\alpha_{2}$, which is a function of the thickness of the three layers $\left(h_{1}, h_{2}\right.$, and $\left.h_{3}\right)$ and the density difference at the two interfaces $\left(\Delta \rho_{1}\right.$ and $\left.\Delta \rho_{2}\right)$. In the first case, the stratification parameters are set to $h_{1}=0.3 H, h_{2}=0.4 H, h_{3}=0.3 H$, and $\Delta \rho_{1}=$ $\Delta \rho_{2}$. The corresponding quadratic nonlinearity coefficient is $\alpha_{2}=2.5 c_{2} / H$. This coefficient represents a convex wave, and the wave amplitude is set to $\eta_{0,2}=0.1 \mathrm{H}$. Additionally, under this special condition, $h_{1}=h_{3}$ and $\Delta \rho_{1}=\Delta \rho_{2}$; this parameter setting is usually applied in laboratory experiments for generating convex waves and makes the mode-1 ISWs vanish. Therefore the quadratic nonlinearity coefficient of mode- 1 ISW is $\alpha_{1} \equiv 0$. The parameters for this case are summarized in Table 1.

Figure 6 shows the convex case solution, from left to right, which are amplitude and horizontal and vertical current components as a function of distance and depth, respectively. First, an elevation wave and a depression wave appear in the upper and lower water columns, respectively (Fig. 6a and c), which indicates that this wave is a convex type. Second, the middle-layer current direction is consistent with the mode-2 ISW propagation direction (Fig. 6b). Therefore, we can use the current direction of the middle layer to determine the propagation direction of the convex wave. The properties of this wave from analytical solutions are analogous to the observation results shown in Fig. 2.

In the second case, the stratification parameters are set to $h_{1}=0.2 \mathrm{H}, h_{2}=0.6 \mathrm{H}, h_{3}=0.2 \mathrm{H}$, and $\Delta \rho_{1}=$ $\Delta \rho_{2}$. The corresponding quadratic nonlinearity coefficient is $\alpha_{2}=-2.5 c_{2} / H$, which represents a concave wave, and the wave amplitude is set to $\eta_{0,2}=-0.1 \mathrm{H}$. Again, the quadratic nonlinearity coefficient of the mode-1 ISW is $\alpha_{1} \equiv$ 0 under this condition, and the mode- 1 ISW vanishes. The parameters of this case are summarized in Table 1.

Figure 7 shows the concave case solution, giving, from left to right, the amplitude and the horizontal and vertical current components as a function of distance and depth, respectively. First, a depression wave and an elevation wave appear in the upper and lower water column, respectively (Fig. 7a and c), demonstrating that the wave behaves as a concave type. Second, the upper- or lower-layer current direction is the same as the wave propagation direction (Fig. 7b). Therefore, the propagation direction of a convex wave is determined by the upper- or lower-layer current direction, suggesting that the concave wave shown in Fig. 3 is propagating to the west. The properties of this wave are different than those for the convex wave but are analogous to the observation results shown in Fig. 3.

Figures 6 and 7 show that the mode-2 ISW polarity depends on the signs of $\eta_{0,2}$ (or $\alpha_{2}$ ). The value of $\alpha_{2}$ is a function of layer thickness $\left(h_{1}, h_{2}\right.$, and $\left.h_{3}\right)$ and the 
Table 1. The parameters for the two analytical three-layer ocean model cases.

\begin{tabular}{lccccccc}
\hline Case & $h_{1}$ & $h_{2}$ & $h_{3}$ & $\delta=\Delta \rho_{1} / \Delta \rho_{2}$ & $\alpha_{2}$ & $\eta_{0,2}$ & Waveform \\
\hline I & $0.3 H$ & $0.4 H$ & $0.3 H$ & 1 & $2.5 c_{2} / H$ & $0.1 H$ & Convex \\
II & $0.2 H$ & $0.6 H$ & $0.2 H$ & 1 & $-2.5 c_{2} / H$ & $-0.1 H$ & Concave \\
\hline
\end{tabular}

(a) $A_{2} / H$

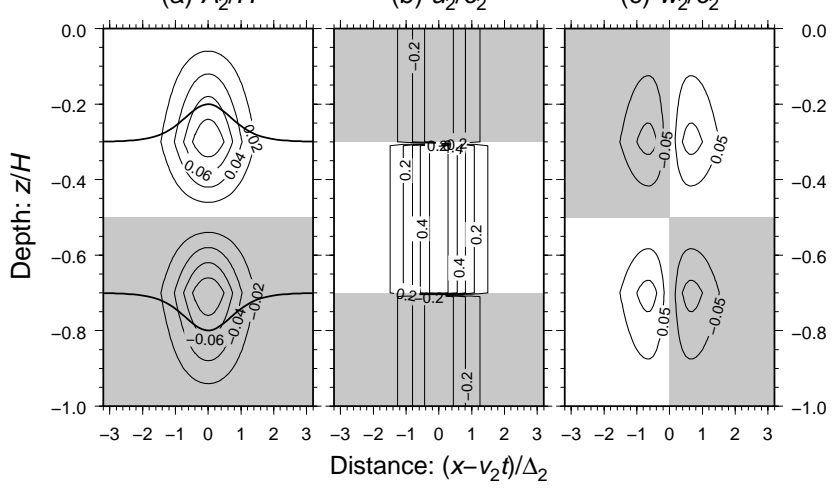

Fig. 6. The left (a), middle (b), and right (c) panels represent the contours of amplitude $\left(A_{2}\right)$ and horizontal $\left(u_{2}\right)$ and vertical $\left(w_{2}\right)$ current components for a convex mode-2 ISW as functions of distance and depth, respectively. The left panel also displays $A_{2}$ at the upper and lower interfaces. All components were nondimensionalized using a horizontal length scale $\Delta_{2}$ (nonlinear characteristic width of a mode-2 ISW), a vertical length scale $H$ (depth), and a speed scale $c_{2}$ (linear phase speed of a mode-2 ISW). Shaded areas show negative values. The contour intervals are 0.02 , 0.1 , and 0.05 for $A_{2}, u_{2}$, and $w_{2}$, respectively.

density differences at the upper and lower interfaces $\left(\Delta \rho_{1}\right.$ and $\Delta \rho_{2}$ ). Figure 8 shows positive $\alpha_{2}$ (convex wave) and negative $\alpha_{2}$ (concave wave) regions as a function of $h_{1}$ and $h_{2}$ when $\delta\left(\Delta \rho_{1} / \Delta \rho_{2}\right)$ was adjusted to $0.5,1$, and 2 , respectively. Most of the region approaches a negative $\alpha_{2}$ or a positive $\alpha_{2}$ when the middle-layer thickness $h_{2}$ becomes larger or smaller, respectively. Different $\delta$ values only slightly influence the patterns in Fig. 8. It follows that the transition of the mode-2 ISW polarity occurs when the middle layer is sufficiently thick or thin. Therefore, the threelayer ocean model implies that a thinner middle layer will be favorable for convex waves, whereas a thicker middle layer would accompany concave waves, which generally agrees with the observations. When $h_{2} / H>0.5, \alpha_{2}$ is always less than zero, meaning that only concave waves are possible. Figures $2 \mathrm{c}$ and $3 \mathrm{c}$ show that the middle-layer thickness (distance between the locus of local $w^{\prime}$ maximum and minimum) of the convex wave $(120 \mathrm{~m})$ was thinner than that of the concave wave $(160 \mathrm{~m})$.

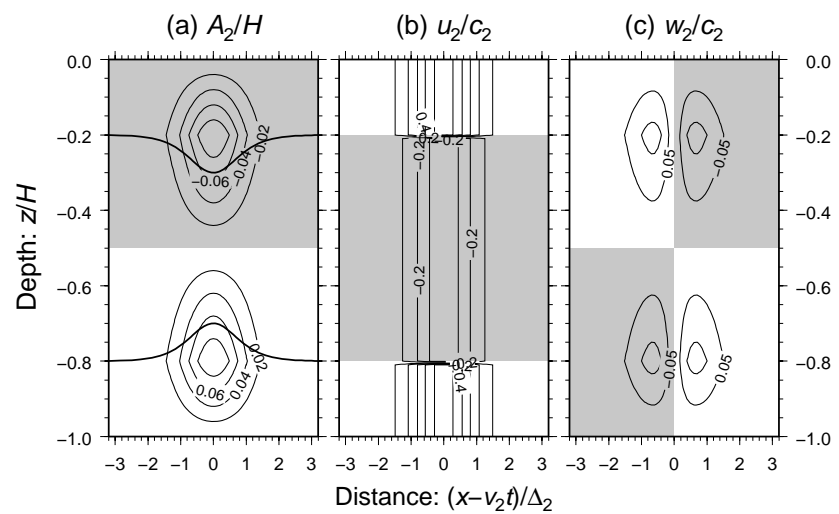

Fig. 7. Same as Fig. 6, except for a concave mode-2 ISW.

According to the analytical solutions, the polarity of mode-2 ISWs is determined by the quadratic nonlinearity coefficient. In a three-layer ocean model, the density difference between the layers slightly influences the quadratic nonlinearity coefficient, and $h_{2}$ mainly affects mode-2 ISW polarity. If the middle-layer thickness is larger than half the water column, the resulting mode-2 ISW waveform is always concave, regardless of the density difference at the two interfaces.

\section{Discussion and summary}

Previous studies have investigated the convex-type wave because the initial condition was always based on an environment with a thin middle layer. Stratification with a "thick" middle layer as defined in Fig. 8 is rare over the continental slope and shelf; thus the concave wave is not commonly observed in the ocean and has not been considered in the laboratory. However, four examples of concave mode-2 ISWs were observed in the northern South China Sea during the VANS/WISE experiment. The three-layer thermal structure with a thick middle layer was sometimes observed over the continental slope and shelf of the northern South China Sea. Figure 4 shows a density profile with a nearly three-layer structure in which the middle-layer thickness was close to half of the water column. This density structure allowed a concave wave to be generated, as suggested by the analytical model results. 
(a)

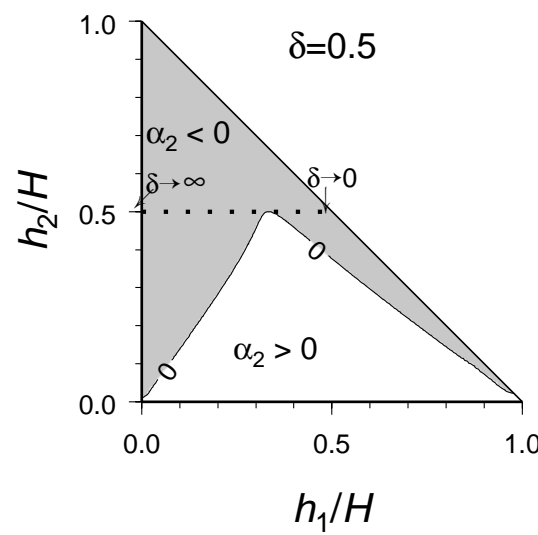

(b)

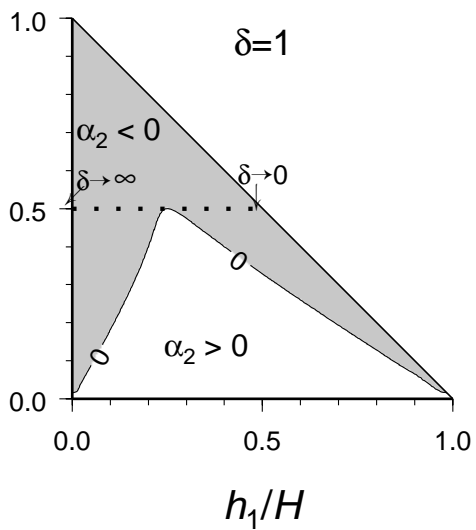

(c)

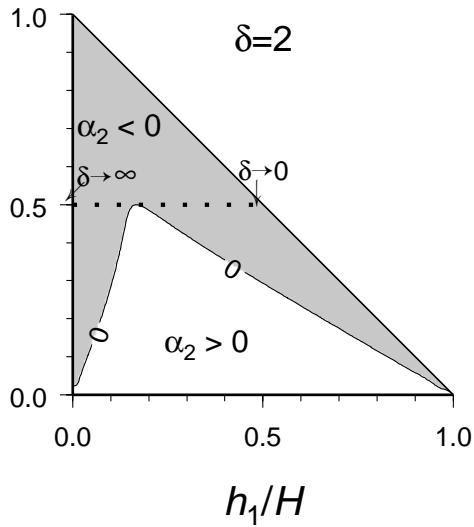

Fig. 8. The solution space for the quadratic nonlinearity coefficient as a function of layer thickness and stratification. The axes have been nondimensionalized by the vertical length scale $H$ (depth). The impact of changing stratification is shown by plotting graphs for different values of the stratification parameter, namely $\delta=\Delta \rho_{1} / \Delta \rho_{2}=0.5,1.0$, and 2.0 in panels (a), (b), and (c), respectively. Shaded areas show negative values. The dashed lines represent the envelope when $\alpha_{2}=0$.

When the resulting mode-2 ISW propagated in the same direction as current in the upper and lower layers, by Eq. (15), a convergence zone $(\partial u / \partial x<0)$ and a divergence zone $(\partial u / \partial x>0)$ were produced in these two layers at the front and rear of the mode-2 ISW, respectively. Due to the rigid-lid boundary condition and volume conservation, the downwelling/upwelling processes had to occur in the upper layer at the front/rear of the wave. Similarly, upwelling/downwelling processes appeared in the lower layer. These processes correspond to a concave-shaped waveform. This result indicates that the concave wave propagated in the same direction as current in the upper and lower layers, respectively. On the other hand, when the resulting mode-2 ISW propagated in the same direction as current in the middle layer, a convergence and a divergence zone were also produced at the front and rear of the mode-2 ISW, respectively, in the middle layer. Consequently, upward/downward and downward/upward displacements were induced at the upper and lower interfaces, respectively, showing a convex wave with a propagation direction that is consistent with the current direction in the middle layer.

The upper layer circulation resembles a mode-1 depression wave for the concave case and a mode- 1 elevation wave for the convex case. This result means that the surface signatures in synthetic aperture radar (SAR) imagery should resemble these waves, but would likely be weaker and more intermittent. The divergence ahead of the convex wave and the convergence behind it should produce a dark/light pattern (smooth/rough) in SAR imagery; the opposite should be true for the concave wave (Alpers, 1985; Liu et al., 1998; Chang et al., 2008). While mode- 2 waves have been observed in moderate resolution imaging spectroradiometer (MODIS) imagery (Yang et al., 2009), they have not yet been observed in SAR imagery.
From the three-layer ocean-model results, the turning point function $\left(\alpha_{2}=0\right)$ is more complicated than that of the two-layer ocean-model results. However, the mode-2 ISW polarities are dependent on layer thicknesses and the density difference at the two interfaces. In particular, the mode-2 ISW waveform is always the concave type as long as the middle-layer thickness is larger than half of the water column. However, this is an analytical three-layer ocean-model result, and thus further studies are needed, especially those that consider more complicated conditions, such as continuous stratification, full nonlinearity, and nonhydrostatic effects.

Using the VANS/WISE mooring observations of the continental slope of the northern South China Sea, data collected by the ADCP, the three RCM8s, and the 16 temperature sensors revealed a number of mode-2 ISW episodes. The mode-2 ISWs had convex-shaped and concave-shaped waveforms, and more convex-shaped episodes occurred than concave-shaped episodes. The vertical structures of mode-2 ISWs generally agreed with the linear modal functions. The maximum/minimum vertical current component loci were close to the nodal points of the horizontal current component. Convex and concave waves were accompanied by thinner and thicker middle layers, respectively. The mode- 2 ISW propagation direction was the same as the current direction in the middle (upper or lower) layer for the convex (concave) wave. The analytical solutions generally agreed with the observations. The mode- 2 ISW polarities are related to the sign of the quadratic nonlinearity coefficient of the K-dV equation. In the three-layer ocean model, the polarities were not only a function of the thickness of each layer but also were related to the density difference at the two interfaces. A convex wave was found when there was a thin middle layer. When the middle-layer thickness was larger than half the 
water column, the resulting mode- 2 ISW was always concave shaped. Although mode-2 ISWs are not as energetic as mode-1 ISWs observed in the same region, they potentially play a significant role in mixing shelf waters (Moum et al., 2008). Future studies are needed to fully elucidate the nature of convex and concave waveforms and their generation, propagation, translation, and dissipation properties.

Acknowledgements. This work was funded by the National Science Council, Taiwan (NSC 93-2611-M-002-019, 93-2611M-012-001, 94-2611-M-002-003, and 94-2611-M-012-001), and the Office of Naval Research. We thank the technical support groups at National Taiwan University and the Naval Postgraduate School, who all worked tirelessly to execute the fieldwork at sea. We are also grateful to the captain and crew members of the $\mathrm{R} / \mathrm{V}$ Ocean Researcher I for their contributions to the success of this experiment.

Edited by: V. I. Vlasenko

Reviewed by: E. Pelinovsky and another anonymous referee

\section{References}

Akylas, T. and Grimshaw, R.: Solitary internal waves with oscillatory tails, J. Fluid Mech., 242, 279-298, 1992.

Alpers, W.: Theory of radar imaging of internal waves, Nature, 314 , 245-247, 1985.

Antenucci, J. P., Imberger, J., and Saggio, A.: Seasonal evolution of basin-scale internal wave field in a large stratified lake, Limnol. Oceanogr., 45, 1621-1638, 2000.

Apel, J. R.: A new analytical model for internal solitons in the ocean, J. Phys. Oceanogr., 33, 2247-2269, 2003.

Apel, J. R., Ostrovsky, L. A., Stepanyants, Y. A., and Lynch, J. F.: Internal solitons in the ocean and their effect on underwater sound, J. Ascust. Soc. Am., 121, 695-722, 2007.

Apel, J. R., Badiey, M., Chiu, C.-S., Finette, S., Headrick, R., Kemp, J., Lynch, J. F., Newhall, A., Orr, M. H., Pasewark, B. H., Tielbuerger, D., Turgut, A., von der Heydt, K., and Wolf, S.: An overview of the 1995 SWARM Shallow-Water Internal Wave Acoustic Scattering Experiment, IEEE J. Oceanic Eng., 22, 465500, 1997.

Benjamin, T. B.: Internal waves of permanent form in fluids of great depth, J. Fluid Mech., 29, 559-592, 1967.

Benny, D. J.: Long non-linear waves in fluid flow, J. Math. Phys., 45, 52-63, 1966.

Boegman, L., Imberger, J., Ivey, G. N., and Antenucci, J. P.: High-frequency internal waves in large stratified lakes, Limnol. Oceanogr., 48, 895-919, 2003.

Bogucki, D. J., Redekopp, L. G., and Barth, J.: Internal solitary waves in the Coastal Mixing and Optics 1996 experiment: Multimodal structure and resuspension, J. Geophys. Res., 110, C02024, doi:10.1029/2003JC002253, 2005.

Chang, M.-H., Lien, R.-C., Yang, Y. J., Tang, T. Y., and Wang, J.: A composite view of surface signatures and interior properties on nonlinear internal waves: Observations and applications, J. Atmos. Ocean. Tech., 25, 1218-1227, 2008.

Davis, R. E. and Acrivos, A.: Solitary internal waves in deep water, J. Fluid Mech., 29, 593-607, 1967.
Duda, T. F., Lynch, J. F., Irish, J. D., Beardsley, R. C., Ramp, S. R., Chiu, C.-S., Tang, T. Y., and Yang, Y. J.: Internal tide and nonlinear internal wave behavior at the continental slope in the northern South China Sea, IEEE J. Oceanic Eng., 29, 1105-1130, 2004

Farmer, D. M. and Smith, J. D.: Tidal interaction of stratified flow with a sill in Knight Inlet, Deep-Sea Res., 27A, 239-254, 1980.

Gear, J. and Grimshaw, R.: A second-order theory for solitary waves in shallow fluids, Phys. Fluids, 26, 14-29, 1983.

Gill, A. E.: Atmosphere-Ocean Dynamics, Academic Press, San Diego, 662 pp., 1982

Grimshaw, R.: Evolution equations for long nonlinear waves in stratified shear flow, Stud. Appl. Math., 65, 159-188, 1981.

Grimshaw, R., Pelinovsky, E., and Talipova, T.: The modified Korteweg - de Vries equation in the theory of large amplitude internal waves, Nonlin. Processes Geophys., 4, 237 250, doi:10.5194/npg-4-237-1997, 1997.

Helfrich, K. R. and Melville, W. K.: Long nonlinear internal waves, Annu. Rev. Fluid Mech., 38, 395-425, 2006.

Honji, H., Matsunaga, N., Sugihara, Y., and Sakai, K.: Experimental observation of internal symmetric solitary waves in a two-layer fluid, Fluid Dyn. Res., 15, 89-102, 1995.

Kao, T. W. and Pao, H.-P.: Wake collapse in the thermocline and internal solitary waves, J. Fluid Mech., 97, 115-127, 1980.

Konyaev, K. V., Sabinin, K. D., and Serebryany, A. N.: Largeamplitude internal waves at the Mascarene Ridge in the Indian Ocean, Deep-Sea Res. Pt. I, 42, 2075-2091, 1995.

Lee, C.-Y. and Beardsley, R. C.: The generation of long nonlinear internal waves in a weakly stratified shear flow, J. Geophys. Res., 79, 453-462, 1974.

Liu, A. K., Chang, Y. S., Hsu, M.-K., and Liang, N. K.: Evolution of nonlinear internal waves in the East and South China Seas, J. Geophys. Res., 103, 7995-8008, 1998.

Maslowe, S. A. and Redekopp, L. G.: Long nonlinear waves in stratified shear flows, J. Fluid Mech., 101, 321-348, 1980.

Maxworthy, T.: On the formation of nonlinear internal waves from the gravitational collapse of mixed regions in two and three dimensions, J. Fluid Mech., 96, 47-64, 1980.

Mehta, A. P., Sutherland, B. R., and Kyba, P. J.: Interfacial gravity currents. II. Wave excitation, Phys. Fluids, 14, 3558-3569, 2002.

Moum, J. N., Nash, J. D., and Klymak, J. M.: Small-scale processes in the coastal ocean, Oceanography, 21, 22-33, 2008.

Ostrovsky, L. A. and Stepanyants, Y. A.: Internal solitons in laboratory experiments: Comparison with theoretical modes, Chaos, 15, 037111, 1-28, 2005.

Pelinovsky, E. N. and Shavratsky, S. K.: Disintegration of cnoidal internal waves in a horizontally inhomogeneous ocean, Atmos. Oceanic Phys., 13, 455-456, 1997.

Ramp, S. R., Yang, Y. J., and Bahr, F. L.: Characterizing the nonlinear internal wave climate in the northeastern South China Sea, Nonlin. Processes Geophys., 17, 481-498, doi:10.5194/npg-17481-2010, 2010.

Rubino, A., Brandt, P., and Weigle, R.: On the dynamics of internal waves in a nonlinear, weakly nonhydrostatic three-layer ocean, J. Geophys. Res., 106, 26899-26915, 2001.

Rusås, P.-O. and Grue, J.: Solitary waves and conjugate flows in a three-layer fluid, Eur. J. Mech. B-Fluids, 21, 185-206, 2002.

Sabinin, K. and Serebryany, A.: Intense short-period internal waves in the ocean, J. Mar. Res., 63, 227-261, 2005. 
Saggio, A. and Imberger, J.: Internal wave weather in a stratified lake, Limnol. Oceanogr., 43, 1780-1798, 1998.

Saggio, A. and Imberger, J.: Mixing and turbulent fluxes in a metalimnion of a stratified lake, Limnol. Oceanogr., 46, 392409, 2001.

Shroyer, E. L., Moum, J. N., and Nash, J. D.: Mode-2 waves on the continental shelf: ephemeral components of the nonlinear internal wave field, J. Geophys. Res., 115, C07001, doi:10.1029/2009JC005605, 2010.

Stamp, A. P. and Jacka, M.: Deep-water internal solitary waves, J. Fluid Mech., 305, 347-371, 1995.

Stastna, M. and Peltier, W.: On the resonant generation of largeamplitude internal solitary waves and solitary-like waves, J. Fluid Mech., 543, 267-292, 2005.

Sutherland, B. R.: Interfacial gravity currents. I. Mixing and entrainment, Phys. Fluids, 14, 2244-2254, 2002.

Terez, D. E. and Knio, O. M.: Numerical simulations of largeamplitude internal solitary waves, J. Fluid Mech., 362, 53-82, 1998.

Tung, K.-K., Chan, T. F., and Kubota, T.: Large amplitude internal waves of permanent form, Stud. Appl. Math., 66, 1-44, 1982.
Vlasenko, V. I.: Multimodal soliton of internal waves, Izv. Atmos. Oceanic Phys., 30, 161-169, 1994.

Vlasenko, V. I. and Hutter, K.: Generation of second mode solitary waves by the interaction of a first mode soliton with a sill, Nonlin. Processes Geophys., 8, 223-239, doi:10.5194/npg-8-223-2001, 2001.

Vlasenko, V. I. and Alpers, W.: Generation of secondary internal waves by the interaction of an internal solitary wave with an underwater bank, J. Geophys. Res., 110, C02019, doi:10.1029/2004JC002467, 2005.

Yang, Y. J., Tang, T. Y., Chang, M.-H., Liu, A. K., Hsu, M.-K., and Ramp, S. R.: Solitons northeast of Tung-Sha Island during the ASIAEX pilot studies, IEEE J. Oceanic Eng., 29, 1182-1199, 2004.

Yang, Y. J., Fang, Y. C., Chang. M.-H., Ramp, S. R., Kao, C.-C., and Tang, T. Y.: Observations of second baroclinic mode internal solitary waves on the continental slope of the northern South China Sea, J. Geophys. Res., 114, C10003, doi:10.1029/2009JC005305, 2009.

Yih, C.-S.: Gravity waves in a stratified fluid, J. Fluid Mech., 8, 481-508, 1960. 